\title{
Management Concentric Collapse of Velopharynx in Obstructive Sleep Apnoea Using a Modified Barbed Palato-Pharyngoplasty Technique
}

\author{
Susan K Sebastian, MS*, Ankur Sharma, MS, Omprakash Chawla, MS and Payal Garg, MS
}

Department of ENT and Head \& Neck Surgery, St. Stephen's Hospital, India

*Corresponding author: Susan K Sebastian, Head of Department of ENT and Head \& Neck Surgery, St. Stephen's Hospital, Tis Hazari, Delhi, India, Fax: +91-11-23932412

\begin{abstract}
Background: Multilevel surgical techniques are important in the treatment of obstructive sleep apnoea especially when the patient is non-compliant to Continuous Positive Airway Pressure therapy (CPAP). A modified pharyngoplasty technique is described in OSA patients where the major cause of upper airway obstruction was due to concentric collapse of velopharynx.

Methods: Twenty four patients with evidence of OSA on polysomnography were included. In all patients the major cause of obstruction was due to concentric collapse of velopharynx as evidenced on Muller Maneuver (MM) and Drug Induced Sleep Endoscopy (DISE). All had non-adherence to (CPAP) therapy and underwent Barbed Palato Pharyngoplasty (BPP) for correction of concentric collapse of velum. Apnoea-Hypopnoea Index (AHI), Lowest Oxygen Saturation (L-sat), Epworth Sleepiness Score (ESS), Visual Analog Score (VAS) for snoring were assessed both pre-operatively and six months post-operatively. All patients underwent awake flexible nasopharyngoscopy with MM to assess the site and score the severity of obstruction before and six months after surgery.
\end{abstract}

Results: Post-operative flexible nasopharyngoscopy with Muller Maneuver revealed absence of collapse of velum in eleven patients, $25 \%$ collapse in 10 and $50 \%$ collapse in 3 patients. Post-operatively, significant reduction of mean values were observed in AHI, ESS, VAS for snoring from 32.04 to $15.27,12.75$ to 4.54 and from 7.17 to 2.54 respectively. The mean L-sat improved from 74.44 to 86.04 . There were no major surgical complications.

Conclusion: BPP is a minimally invasive knotless technique and is best suited in OSA, where the major obstruction is due to concentric collapse of the velum.

\section{Keywords}

Obstructive sleep apnoea, Concentric collapse of velum, Barbed surgery, Palatopharyngeus muscle, Muller maneuver

\begin{abstract}
Abbreviations
OSA: Obstructive Sleep Apnoea; BPP: Barbed Palato-Pharyngoplasty; MM: Mueller Maneuver; DISE: Drug Induced Sleep Endoscopy; CPAP: Continuous Positive Airway Pressure; AHI: Apnoea-Hypopnoea Index; L-SAT: Lowest Oxygen Saturation; UPPP: Uvulopalatopharyngoplasty; ESP: Expansion Sphincter Pharyngoplasty; BRP: Barbed Reposition Pharyngoplasty
\end{abstract}

\section{Introduction}

Obstructive Sleep Apnoea (OSA) is a common health problem that is associated with potentially serious consequences [1] and results from single or multiple levels of obstruction of the upper airway. Although Continuous Positive Airway Pressure (CPAP) therapy is an effective mode of treatment, itseffectiveness is often limited by low patient compliance [2]. Surgical treatment aims at relieving the pharyngeal collapsibility and multilevel surgery is increasingly being recommended in the management of OSA. Uvulopalatopharyngoplasty (UPPP) [3] which used to be the most popular surgery for OSA, addresses palatopharyngeal obstruction but has a relatively low success rate [4]. It has fallen out of favour, because it failed to address the multilevel obstruction and involved resection of a major portion of the soft palate which in turn lead to severe postoperative scar-

Citation: Sebastian SK, Sharma A, Chawla O, Garg P (2020) Management Concentric Collapse of Velopharynx in Obstructive Sleep Apnoea Using a Modified Barbed Palato-Pharyngoplasty Technique. J Sleep Disord Manag 6:028. doi.org/10.23937/2572-4053.1510028

Accepted: July 02, 2020: Published: July 04, 2020

Copyright: (C) 2020 Sebastian SK, et al. This is an open-access article distributed under the terms of the Creative Commons Attribution License, which permits unrestricted use, distribution, and reproduction in any medium, provided the original author and source are credited. 
ring and poor long-term outcome. In the last decade, many new palatal surgical techniques have evolved that address mainly the lateral pharyngeal wall with an aim to enlarge laterally the oropharyngeal inlet. These procedures include lateral pharyngoplasty [5], Z-palatoplasty [6], Uvulopalatoplasty [7], expansion sphincter pharyngoplasty (ESP) [8] and relocation pharyngoplasty [9]. Among these, ESP proved to be superior to UPPP. Viccini, et al. has introduced Barbed Relocation Pharyngoplasty [10] where the lateral insertion of the soft palateis relocated to increase the retropalatal airway.In addition, they have used barbed suturing techniques and the relocation is stabilised on pterygomandibular raphe.

Different types of anterior palatoplasty techniques have shown encouraging results in the correction of antero-posterior collapse of palate [11-13]. Mantowani described the "Roman Blinds" technique that lifts up and anchors the soft palate to stable structures [14] which improved the stability of the anterior palatal reconstruction.

Multilevel surgery includes multiple surgical techniques according to the sites and pattern of obstruction [15-17]. It has been observed that velum is one of the most frequent sites of obstruction [16] and the degree of collapse of the airway at velopharynx can be individually assessed by awake nasopharyngoscopy with Muller Maneuver (MM) [18]. Hence a comparison between pre-operative and post-operative MM can help us to evaluate the benefit following surgery at the velum.

Drug Induced Sleep Endoscopy (DISE) evaluates the pattern and severity of airway collapse during sleep. Pattern of collapse can be lateral, anteroposterior or concentric [19]. Although lateral or anteroposterior collapse alone is more common, patients with a thick hanging palate can have a combination of both, resulting in concentric collapse of the velum. Surgical treatment of concentric collapse is more demanding and should address both antero-posterior and lateral collapse to attain optimum results [20].

The present study is designed to address the effect of a minimally resective barbed surgical technique, Barbed Palato Pharyngoplasty (BPP) that can address the obstruction due to concentric collapse of the velopharynx. Following multilevel surgery, it is difficult to assess the contribution of each technique to the ultimate result of the procedure using polysomnography parameters alone.In the current study, awake naspharyngoscopy with Muller Maneuver (MM) is also used for evaluation of the procedure.

\section{Material and Methods}

This is a retrospective study of twentyfour patients with predominant concentric obstruction at thevelum who underwent BPP as a stand-alone or as part of multilevel surgery for OSA at St. Stephen's Hospital, Delhi, India from May 2017 to July 2018.
Complete clinical evaluation including assessment of Epworth Sleepiness Score (ESS) [21] and Visual Analog Score (VAS) [20] for snoring was done in all patients. Apnoea-Hypopnoea Index ( $\mathrm{AHI}$ ) and lowest oxygen saturation level (L-sat) were assessed by polysomnography [21]. Awake naspharyngoscopy with Muller Maneuver (MM) was done pre-operatively. Collapse during MM was rated on a five-point scale, from 0 to 4 , in which 0 is no collapse, 1 is approximately $25 \%$ collapse, 2 is approximately $50 \%$ collapse, 3 is approximately $75 \%$ collapse, and 4 is complete collapse [18]. The grade and patterns of upper airway collapse during DISE were evaluated and recorded with the VOTE classification system [19].

\section{Selection criteria}

- Evidence of OSA on Polysomnography [22].

- Non-compliance to CPAP.

- Patients with concentric collapse of velum as the major siteof obstruction on awake nasopharyngoscopy with MM score of 3 or 4 for palate.

- MM score two or less for other sites.

Out of 77 patients who underwent multilevel sleep surgery during this period, 24 patients satisfied the above criteriaand were included in this study. All patients were reassessed with MM scoring, Polysomnography with $\mathrm{AHI}$ and L-sat, ESS and VAS for snoring at six months after surgery.

This study received the approval from the institutional ethics committee (SSHEC/R0017 dated 14/8/2018).

\section{Surgical procedure}

Oropharynx is exposed using Boyle-Davis mouth gag. Palatopharyngeus muscle is exposed by doing an intra-capsular tonsillectomy. Palatopharyngeus muscle is partially dissected and freed on its lateral border. The upper part of the palatopharyngeus, where it runs above the upper pole of the tonsil, is exposed by making parauvular cuts using bipolar cautery. Extra effort is made to expose the maximum length of palatopharyngeus and its medial border is preserved with the mucosal cover intact. Uvula is also left undisturbed.

Posterior nasal spine in the midline and pterygoid hamuli and pterygomandibular raphes are identified on both sides. A central rectangular area, $3 \mathrm{~cm}$ in length by 1 $\mathrm{cm}$ in width is marked on the anterior surface of the soft palate. Mucosa and sub-mucosal tissues are removed using bipolar cautery exposing the underlying muscle. Using a 3/0 polydioxane bidirectional barbed suture 24 $\times 24 \mathrm{~cm}$ in length with two needles (Stratafix) suturing is started in the midline on the right, close to the posterior nasal spine. Through the soft palate musculature the suture is directed to reach the midline lower border of the rectangular denuded area. From the midpoint, three or four sutures are made laterally between the upper and 


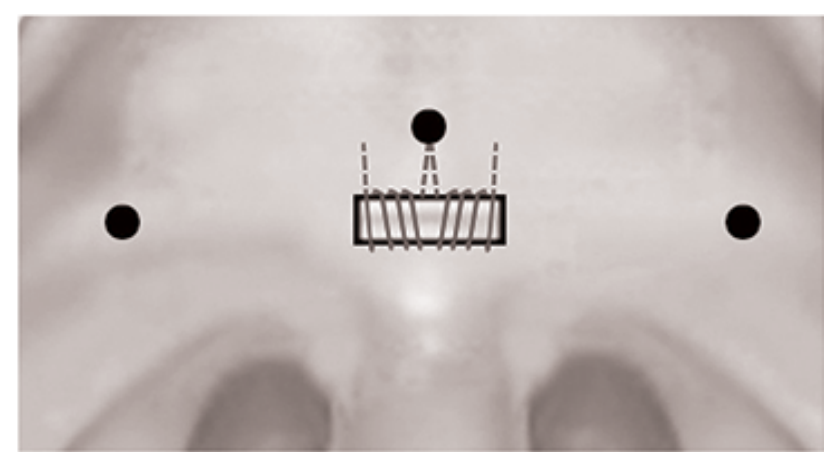

Figure 1: Central black dot: Posterior nasal spine; Lateral black dots: Pterygoid hamulus. Three or four sutures were made approximating the upper and lower borders of the denuded area. From the upper lateral corners of the rectangle sutures were taken to the palatal aponeurosis.

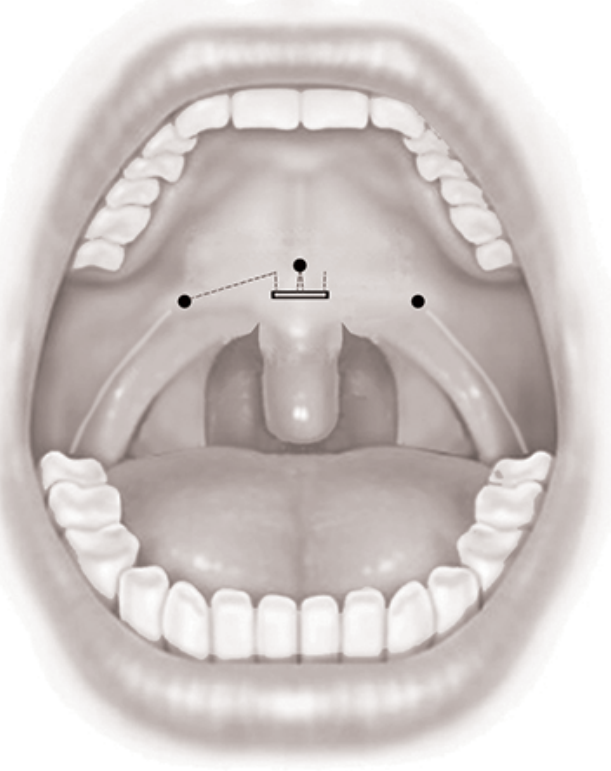

Figure 2: The end of the suture is taken close to the right hamulus.

lower borders of the denuded area, gathering the exposed muscle and tightening it until the needle reaches the right upper corner of the rectangle. From the upper corner, suture is made to exit at the palatal aponeurosis. The same procedure is repeated on the left half of the palate with the transition point of the barbed suture at the level of posterior nasal spine (Figure 1).

Lateral pharyngeal wall repair is done using the free ends of the barbed suture on either side. The free end is made to exit close to the pterygoid hamulus by taking the suture through the musculature (Figure 2). Multiple such sutures are applied between the pterygomandibular raphe and the palatopharyngeus and then tightened. An extended lateral pharyngoplasty is achieved by including the upper and lower most ends of the Palatopharyngeus in the sutures. In patients with significant lateral wall collapse, multiple loops are also taken around the Palatopharyngeus muscle, during each pass between the muscle and pterygomandibular raphe. The

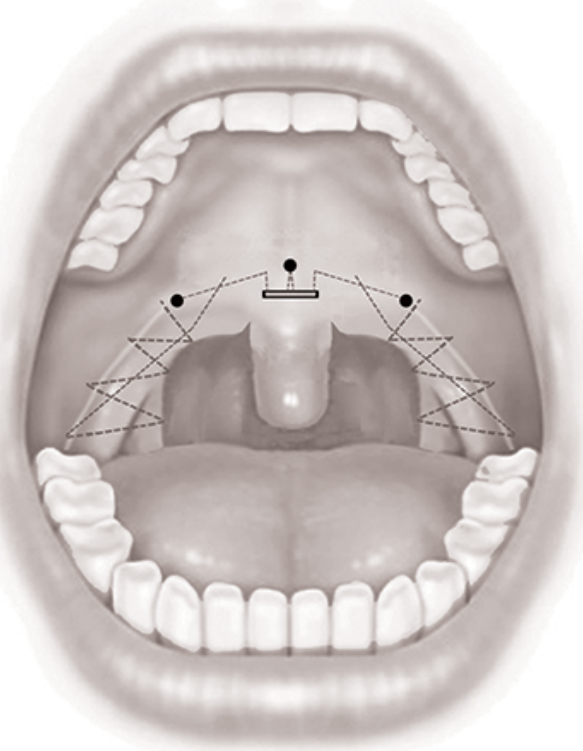

Figure 3: Multiple sutures taken between the pterygomandibular raphe and palatopharyngeus muscles on both sides.

same procedure is repeated on the left side using the other end of the barbed suture (Figure 3 ).

\section{Statistical analysis}

Statistical analysis was performed using paired t-test to compare the preoperative and six months postoperative sleep parameters. P-value less than 0.05 considered as significant at $95 \%$ confidence level. The statistical software SPSS version 18.0 was used in the analysis.

\section{Results and Analysis}

The study included 16 men and 8 women with a mean age of $43.96 \pm 11.21$ years (Table 1 ). BMI ranged from 22 to $36.73 \mathrm{~kg} / \mathrm{m}^{2}$. (Mean $28.77 \pm 4.99$ ) Average neck circumference was $39.6 \mathrm{cms}$. Three patients had mild, eleven had moderate and nine had severe OSA according to AHI values on polysomnography [8]. Awake nasopharyngoscopy with $\mathrm{MM}$ revealed concentric collapse of palate in all patients with a score of three or four, indicating a collapse of more than $75 \%$, while collapse at other sites were two or less. During DISE, velum was identified as the major site of collapse in all patients.

In addition to BPP, other multilevel surgical procedures were also carried out to correct lesser degrees of collapse of other sites in nine patients; endoscopic midline glossectomy-6, thyrohyoidopexy-3, and suprahyoid partial epiglottectomy-1.

Re-evaluation of airway was done six months after surgery using awake nasopharyngoscopy with MM. Post-operative MM revealed complete resolution of collapse of palate in eleven, $25 \%$ collapse in ten and $50 \%$ collapse in three patients (Table 1 ).

Evaluation of sleep parameters showed reduction of mean AHI value from 32.04 to 15.27 , mean ESS scores from 12.75 to 4.54 VAS score for snoring from 7.17 to 2.54 
离

3

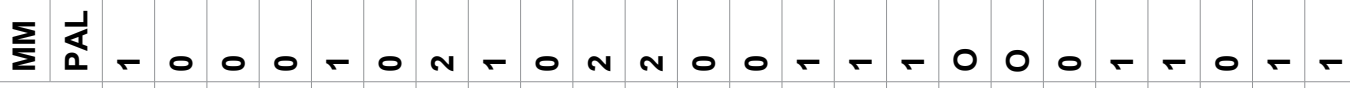

芠

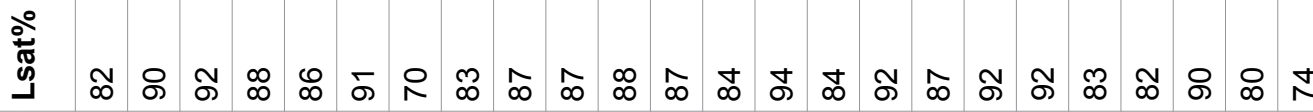

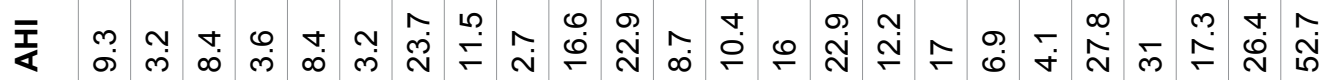
竞

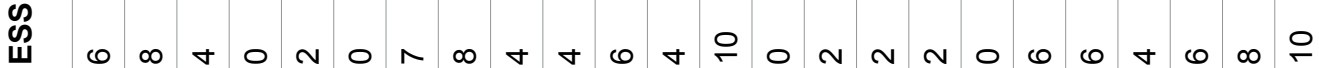

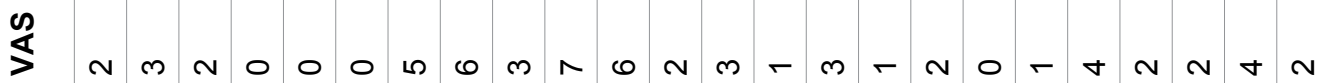

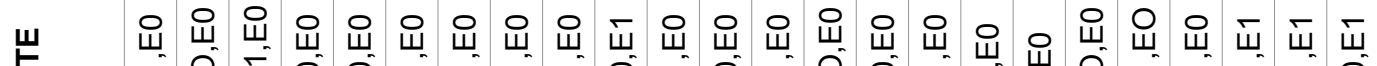

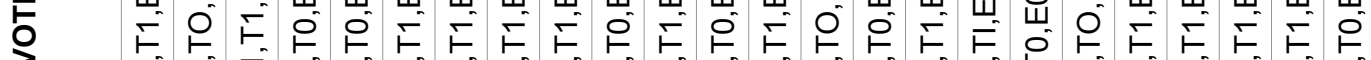
出

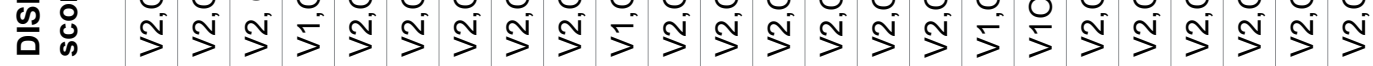

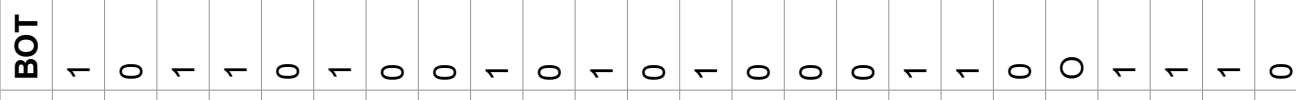

3

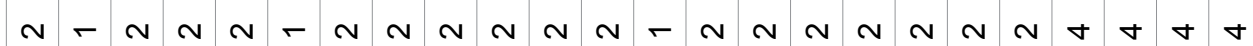

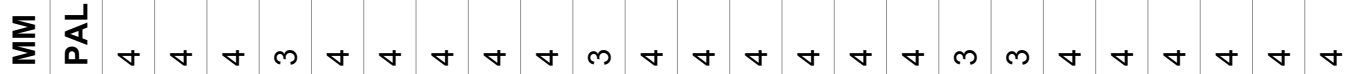

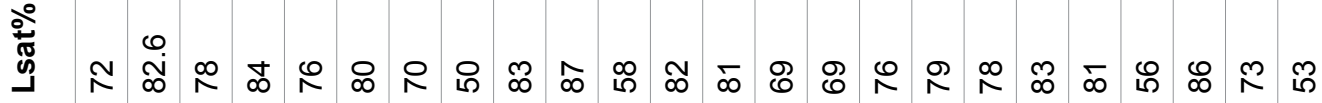

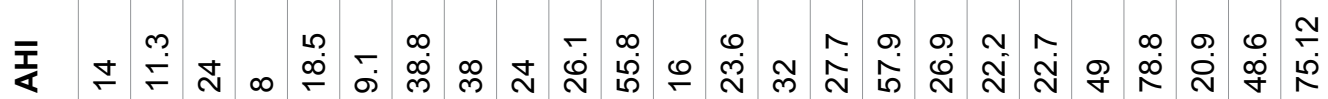
\}

岂

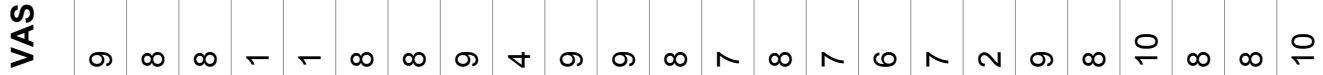

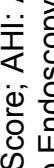
की बे

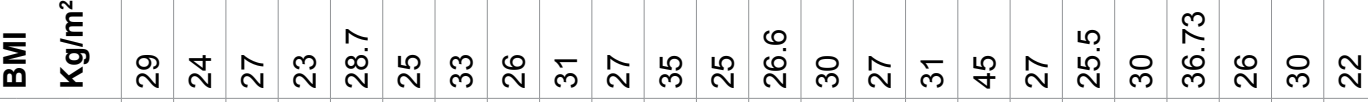

\& $\quad$ 守 $\frac{0}{2} \frac{\pi}{0}$

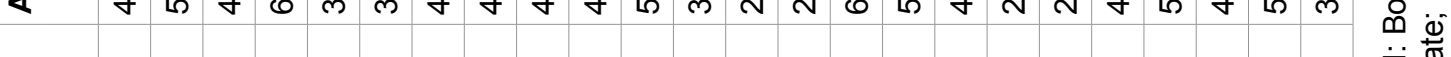
கீ人 
Table 2: Pre- and post-operative sleep parameters.

\begin{tabular}{|l|l|l|l|l|l|}
\hline Sleep parameters & Mean & N & Standard Deviation & t-value & p-value \\
\hline Pre-VAS & 7.17 & 24 & 2.582 & 9.695 & $<0.001$ \\
\hline Post-VAS & 2.54 & 24 & 1.978 & & \\
\hline Pre-ESS & 12.75 & 24 & 5.067 & 9.595 & $<0.001$ \\
\hline Post-ESS & 4.54 & 24 & 3.107 & & $<0.001$ \\
\hline Pre-AHI & 32.04 & 24 & 19.570 & 6.83 & $<0.001$ \\
\hline Post AHI & 15.29 & 24 & 11.660 & & $<.048$ \\
\hline Pre-Lsat & 74.44 & 24 & 10.582 & & $<$ \\
\hline Post-Lsat & 86.04 & 24 & 5.790 & & $<$ \\
\hline
\end{tabular}

VAS: Visual Analog Score for snoring; ESS: Epworth Sleepiness Score; AHI: Apnoea Hypopnoea Index; LSAT: Lowest Oxygen Saturation; N: Number of Patients; Pre: Preoperative; Post: Postoperative

and an increase of L-sat from 74.44 to 86.04 (Table 2).

In the first 48 hours of post-operative period, all patients had pain and dysphagia which responded well to non-sedating analgesics. Seven patients had persistent pain lasting for one week (29\%). Two patients had mucosal granulations at the palatoplasty site (8\%), which eventually healed. Extrusion of pieces of suture material was experienced in eight cases (33.3\%). None of the patients had post-operative bleeding, velopharyngeal incompetency or difficulty in clearing the postnasal secretions.

\section{Discussion}

OSA is commonly associated with multilevel obstruction requiring multilevel surgical corrections [15$17,22,23]$, although obstruction at certain anatomical levels may contribute more towards the severity of OSA $[16,17]$. Targeting the major levels of obstruction is of vital importance in the success of multilevel surgery [16]. In the current series, we confined our selection to patients with significant concentric collapse of the velum, with partial or no collapse of other sites (Table 1). Correction of concentric collapse of velum requires a combination of lateral pharyngoplasty and anterior palatoplasty [7]. The modified pharyngoplasty technique, BPP, primarily addresses concentric collapse of the velum. Hence a favourable surgical outcome will reflect the success of the new technique.

Lateral pharyngeal wall procedures like Expansion sphincter pharyngoplasty (ESP) introduced by Pang and Woodson [8] and its modifications [9], are effective in dealing with lateral collapse of the velum but have the disadvantage of disrupting the muscle mass and its normal anatomy. BPP is inspired by BRP and is similar to it as far as the lateral pharyngoplasty part is concerned. The main differences between BRP and BPP are:

- We have tried to restore the function and anatomy of the palatopharyngeus especially in the region of the velum. Palatoharyngeus exhibits respiratory activity and factors that compromise its upper airway dilating effect can lead to retropalatal collapse [24]. In severe cases of OSA, the compromise of upper air- way dilating effect of palatopharyngeus muscle becomes a significant factor contributing to the lateral collapse. Considering this fact, we have taken measures to expose and include the maximum length of palatopharyngeus that can be reached through the oropharynx, differing from the previously described procedures. Using multiple loops of suture, we ensured stiffening and effective lateralisation of palatopharyngeus.

- The major difference is in the fact that we have addedan anterior palatoplasty to the lateral pharyngoplasty procedure that improved the antero-posterior dimensions of the velum as well. This part of the reconstruction is stabilised on the posterior nasal spine and the palatal aponeurosis.

Following the procedure, soft palate assumed a superior as well as anterior position, enlarging the retropalatal and oropharyngeal spaces in an antero-posterior and superior direction. The extended lateral repositioning technique of the palatopharyngeus has resulted in improved side to side dimensions. The use of self-locking, reabsorbable sutures in pharyngoplasty procedures has helped in stiffening the airway and allowed preservation of muscles including palatopharyngeus without interfering with their function [21-23]. We have preserved the uvula also, ensuring its anatomy and function. BPP combines the benefits of an extended lateral pharyngoplasty, anterior palatoplasty and barbed suturing technique. It has opened up the velum and oropharynx in all three dimensions. Hence significant improvement was observed in concentric collapse, even in patients with moderate to severe obstruction.

Muller Maneuver scoring has been shown to be a reliable method in assessing the site and degree of collapse of the upper airway and has comparable efficacy as with DISE [25]. Post-operative nasopharyngoscopy with $\mathrm{MM}$ has revealed significant reduction in the severity of collapse at the velum in all the patients. Other procedures done as parts of multilevel surgery in these patients do not influence the results of MM at the velum. Hence the stability of velum indicates the effectiveness of BPP. 
Although there are several barbed oropharyngeal surgeries described in the recent literature $[10,26]$ there is only limited data on barbed techniques addressing concentric collapse of the velum and oropharynx. The Alianza technique by Mantiowani, is effective in mild to moderate cases of OSA with concentric collapse of the velum [27], but this study does not include severe cases. In our series, in spite of including patients with severe OSA, we have experienced encouraging results in terms of improvement in AHI, ESS and VAS for snoring.

Our study also has certain limitations. Being a multilevel procedure it is difficult to quantify the contribution of other surgical procedures to the final outcome on polysomnography parameters. We have observed this limitation in other studies on multilevel OSA surgeries as well $[17,27]$. We have tried to overcome this limitation by selecting agroup of patients who have predominant collapse of the velum and by including MM evaluation in the post-operative evaluation.

\section{Keypoints}

- Concentric collapse is the result of antero-posterior and lateral collapse at the velum.

- BPP combines the benefits of an extended lateral pharyngoplasty, anterior palatoplasty and barbed suturing technique [7-9] and opens up the velum in all three dimensions.

This technique is minimally invasive and knotless with no disruption of muscles and restores the functional anatomy of the velum.

Authors have no financial disclosure or conflicts of interest.

\section{Ethics Statement/Confirmation of Patient's Con- sent}

Ethics approval was obtained from the institutional review board. The patient's consent was obtained for the procedure.

\section{References}

1. Young T, Peppard PE, Gottlieb DJ (2002) Epidemiology of obstructive sleep apnea: A population health perspective. Am J Respir Crit Care Medicine 165: 1217-1239.

2. Weaver TE, Grunstein RR (2008) Adherence to continuous positive airway pressure therapy: The challenge to effective treatment. Proc Am Thorac Soc 5: 173-178.

3. Fujita S, Conway W, Zorick F, Roth T (1981) Surgical correction of anatomic abnormalities in obstructive sleep apnoea syndrome: Uvulopalatopharyngoplasty. Otolaryngol Head Neck Surg 89: 923-934.

4. Soares D, Sinawe H, Folbe AJ, Yoo G, Badr S, et al. (2012) Lateral oropharyngeal wall and supraglottic airway collapse associated with failure in sleep apnea surgery. Laryngoscope 122: 473-479.

5. Cahali MB (2003) Lateral pharyngoplasty: A new treatment for obstructive sleep apnea hypopnea syndrome. Laryngoscope 113: 1961-1968.
6. Friedman M, Ibrahim HZ, Vidyasagar R, Pomeranz J, Joseph NJ (2004) Z-palatoplasty (ZPP): A technique for patients without tonsils. Otolaryngol Head Neck Surg 131: 89-100.

7. Friedman $M$, Ibrahim $H$, Lowenthal $S$, Vidyasagar $R$, Joseph NJ (2004) Uvulopalatoplasty (UP2): A modified technique for selected patients. Laryngoscope 114: 441-449.

8. Pang KP, Woodson BT (2007) Expansion sphincter pharyngoplasty: A new technique for the treatment of obstructive sleep apnea. Otolaryngol Head Neck Surg 137: 110-114.

9. Li HY, Lee LA (2009) Relocation pharyngoplasty for obstructive sleep apnea. Laryngoscope 119: 2472-2477.

10. Vicini C, Hendawy E, Campanini A, Eesa M, Bahgat A, et al. (2015) Barbed reposition pharyngoplasty (BRP) for OSAHS: A feasibility, safety, efficacy and teachability pilot study. "We are on the giant's shoulders". Eur Arch Otorhinolaryngol 272: 3065-3070.

11. Mair EA, Day RH (2000) Cautery-assisted palatal stiffening operation. Otolaryngol Head Neck Surg 122: 547-556.

12. Pang KP, Terris DJ (2007) Modified cautery-assisted palatal stiffening operation: New method for treating snoring and mild obstructive sleep apnea. Otolaryngol Head Neck Surg 136: 823-826.

13. Pang KP, Tan R, Puraviappan P, Terris DJ (2009) Anterior palatoplasty for the treatment of OSA: Three year results. Otolaryngol Head Neck Surg 141: 253-256.

14. Mantovani M, Minetti A, Torretta S, Pincherle A, Tassone $G$, et al. (2012) The velo-uvulo-pharyngeal lift or 'roman blinds' technique for treatment of snoring: A preliminary report. Acta Otorhinolaryngol Ital 32: 48-53.

15. Ishman SL, Ishii LE, Gourin CG (2014) Temporal trends in sleep apnea surgery: 1993-2010. Laryngoscope 124: 12511258.

16. Phua CQ, Yeo WX, Su C, Mok PKH (2017) Multilevel obstruction in obstructive sleep apnoea: Prevalence, severity and predictive factors. J Laryngol Otol 131: 982-986.

17. Lin HC, Friedman M, Chang HW, Gurpinar B (2008) The efficacy of multilevel surgery of the upper airway in adults with obstructive sleep apnea/hypopnea syndrome. Laryngoscope 118: 902-908.

18. David J Terris, Matthew M Hanasono, Yung C Liu (2000) Reliability of the Muller Maneuver and its association with sleep-disordered breathing. Laryngoscope 110: 1819-1823.

19. Kezirian EJ, Hohenhorst W, de Vries N (2011) Drug-induced sleep endoscopy: The VOTE classification. Eur Arch Otorhinolaryngol 268: 1233-1236.

20. Pang KP, Piccin O, Pang EB, Pang KA, Chan YH, et al. (2016) Combined expansion pharyngoplasty and anterior palatoplasty for the treatment of OSA. Indian J Otolaryngol Head Neck Surg 68: 528-533.

21. Vishesh K Kapur, Dennis H Auckley, Susmita Chowdhuri, David C Kuhlmann, Reena Mehra, et al. (2017) Clinical practice guideline for diagnostic testing for adult obstructive sleep apnea: An American Academy of Sleep Medicine Clinical Practice Guideline. J Clin Sleep Med 13: 479-504.

22. Riley RW, Powell NB, Guilleminault C (1993) Obstructive sleep apnea syndrome: A surgical protocol for dynamic upper airway reconstruction. J Oral Maxillofac Surg 51: 742-747.

23. Friedman M, Lin HC, Gurpinar B, Joseph NJ (2007) Minimally invasive single-stage multilevel treatment for obstructive sleep apnea/hypopnea syndrome. Laryngoscope 117: 1859-1863. 
24. Abdelrahman EM Ezzat, Hanna M El-Shenawy (2015) Palatopharyngeus the missing palatal muscles: Anatomical and physiological review. Ann Maxillofac Surg 5: 226-228.

25. Danny Soares, Adam J Folbe, George Yoo, M Safwan Badr, James A Rowley, et al. (2013) Drug-induced sleep endoscopy vs awake müller's maneuver in the diagnosis of severe upper airway obstruction. Otolaryngol Head Neck Surg 148: 151-156.
26. Vicini C, Meccariello G, Cammaroto G, Rashwan M, Montevecchi $F$ (2017) Barbed reposition pharyngoplasty in multilevel robotic surgery for obstructive sleep apnoea. Acta Otorhinolaryngol Ital 37: 214-217.

27. Mantovani M, Carioli D, Toretta S, Rinaldi V, Ibba T, et al. (2017) Barbed snore surgery for concentric collapse at the velum: The Alianza technique. J Craniomaxillofac Surg 45: $1794-1800$ 